\title{
Comparison of continuous femoral nerve block (CFNB/SA) and continuous femoral nerve block with mini-dose spinal morphine (CFNB/SAMO) for postoperative analgesia after total knee arthroplasty (TKA): a randomized controlled study
}

Petchara Sundarathiti, Jadesadha Thammasakulsiri, Supawadee Supboon, Supalak Sakdanuwatwong and Molruedee Piangjai

\begin{abstract}
Background: Unsatisfactory analgesia for major knee surgery with femoral nerve block (FNB) alone was reported and the additional benefit of sciatic block to continuous femoral nerve block (CFNB) was not conclusive. The aim of the present study was to find the benefit of the additional mini-dose spinal morphine $(0.035 \mathrm{mg})$ to CFNB for postoperative pain control and to compare their associated side effects after total knee arthroplasty (TKA).

Methods: After written informed consent and with Institutional Ethics Committee approval, 68 American Society of Anesthesiologists (ASA) Physical Status I-III patients scheduled for elective unilateral TKA under spinal anesthesia $(S A)$ were included in the present prospective, randomized controlled study. The patients were allocated into two groups. CFNB was placed in all patients by the inguinal paravascular approach with $20 \mathrm{ml}$ of $0.25 \%$ levobupivacaine. Group I (named CFNB/SA group), SA was administered with $2.8 \mathrm{ml}$ levobupivacaine and Group II (named CFNB/SAMO group), SA with $2.8 \mathrm{ml}$ levobupivacaine plus morphine $0.035 \mathrm{mg}$. At Post Anesthesia Care Unit (PACU), pain and other adverse effects were recorded. Pain was assessed by visual analog scale (VAS) 0-10. Tramadol $50 \mathrm{mg}$ intravenous (IV) was given if the VAS $>4$. In the ward, all patients were maintained by continuous femoral infusion of $0.125 \%$ levobupivacaine rate $7 \mathrm{ml} / \mathrm{hr}$ and then reduced to $5 \mathrm{ml} / \mathrm{hr}$ if VAS $\leq 3$.

Results: Patient's demographics data in each group were not different. At post-operative (PO) 12-24 h, the VAS scores were significantly lesser in the CFNB/SAMO group. Cumulative tramadol IV requirement for PO48h were also significantly lesser in the CFNB/SAMO group. Nausea, vomiting and numbness were significantly greater in the CFNB/ SAMO group during early postoperative period (PO1-6 h).
\end{abstract}

Conclusion: Though in some patients CFNB was inadequate, a mini-dose of intrathecal morphine $(0.035 \mathrm{mg})$ in addition to CFNB was found to be effective with minimal side effects.

Trial registration: Thai Clinical Trial Registry (identifier: TCTR20150609003, date of registration: 6 June 2015).

Keywords: Continuous femoral nerve block (CFNB), Total knee arthroplasty (TKA), Levobupivacaine, Mini-dose spinal morphine, Postoperative analgesia

\footnotetext{
* Correspondence: petcharas@gmail.com

Department Of Anesthesiology, Ramathibodi Hospital, Mahidol University,

Bangkok, Thailand
}

CiöMed Central
C 2016 Sundarathiti et al. Open Access This article is distributed under the terms of the Creative Commons Attribution 4.0 International License (http://creativecommons.org/licenses/by/4.0/), which permits unrestricted use, distribution, and reproduction in any medium, provided you give appropriate credit to the original author(s) and the source, provide a link to the Creative Commons license, and indicate if changes were made. The Creative Commons Public Domain Dedication waiver (http://creativecommons.org/publicdomain/zero/1.0/) applies to the data made available in this article, unless otherwise stated. 


\section{Background}

A large number of patients who undergo knee surgery experience moderate to severe postoperative pain that interferes with participation in early physical therapy [1-3]. Severe postoperative pain can contribute to immobilityrelated complications, delay in hospital discharge, and interfere with functional outcome [4,5]. Multiple techniques of postoperative pain control have been used after total knee arthroplasty (TKA). Previous studies comparing peripheral nerve block (PNB) with epidural analgesia (EA) for major knee surgery have demonstrated comparable analgesia and improvement in side-effect profile associated with PNB [6, 7].

The femoral nerve along with contributions from the sciatic and obturator nerves at the posterior and the medial aspects respectively, provide sensory innervations of the knee. These three terminal nerves are targeted by PNB techniques for major knee surgery $[6,8-10]$. Reports of satisfactory analgesia with femoral nerve block (FNB) alone $[2,3]$ are countered by studies that found it to be inadequate [11-13]. Sundarathiti $\mathrm{P}$ et al. reported a lesser analgesia efficacy at postoperative (PO) 6-12 $\mathrm{h}$ in continuous FNB (CFNB) group compared with epidural analgesia [7]. Though sciatic blocks analgesic effect is undisputed, there are conflicting opinions about its general benefits in light of the additional time, costs and required skill of therapists $[6,10]$.

In response of surgeons' concerns regarding postoperative sciatic block (e.g, difficulty in diagnosing peroneal nerve injury or an evolving sciatic nerve injury from compartment syndrome), we attempted to limit its use. The aim of the present study was to find the effect of adding a mini-dose spinal morphine $0.035 \mathrm{mg}$ (based on our pilot study) to CFNB for postoperative pain control in patients with total knee arthroplasty (TKA).

\section{Ethical consideration}

This study was conducted according to the declaration of Helsinki. Informed consent was obtained and documented from the participants before data collection. The final protocol and written informed consent form had been approved by Ethics Committee, Faculty of Medicine Ramathibodi Hospital, Mahidol University. This study has been registered at Thai Clinical Trial Registry (identifier: TCTR 20150609003).

\section{Methods}

After written informed consent and with Institutional Ethics Committee approval from June 2012 to June 2015, 70 American Society of Anesthesiologists (ASA) physical status I-III patients scheduled for elective unilateral TKA under spinal anesthesia (SA) were included in the prospective, randomized controlled blind study.
Exclusion criteria included age $<40$ years or $>80$ years, body mass index $(\mathrm{BMI})>45$, renal insufficiency [Creatinine level $(\mathrm{Cr})>1.5 \mathrm{mg} / \mathrm{dl}]$, pre-existing neurological deficit, inability to comprehend pain scales, chronic opioid use, and contraindications to either neuraxial block or FNB. The patients were allocated into two parallel groups at a ratio of 1:1, using random number table. All patients were premedicated with oral lorazepam $0.5 \mathrm{mg}$ $1 \mathrm{~h}$ before surgery and were sedated with midazolam $1 \mathrm{mg}$ and fentanyl $25 \mathrm{mcg}$ intravenously before conducting anesthesia. CFNB was placed in all patients by the inguinal paravascular approach, $19 \mathrm{G}, 50 \mathrm{~mm}$ needle (PAJUNK ${ }^{\oplus}$, PlexoLong NanoLine acc, Meier, Germany). Localized femoral nerve was defined by quadriceps twitch at $<0.5 \mathrm{mAmp}$ using a stimulation of $0.1 \mathrm{~ms}$ at $2 \mathrm{~Hz}$. After negative aspiration, $20 \mathrm{ml}$ of $0.25 \%$ levobupivacaine was administrated and catheter was inserted 3-4 $\mathrm{cm}$ past the cannula. SA was done in lateral position at lumbar vertebrae segment (L) 3-4, 27-G needle. Group I (named CFNB/SA), received SA with $2.8 \mathrm{ml}$ levobupivacaine. Group II (named CFNB/SAMO), received SA with $2.8 \mathrm{ml}$ levobupivacaine plus morphine $0.035 \mathrm{mg}$. Urinary catheters were placed in all patients and were continued until $24 \mathrm{~h}$ post-operative.

The standard monitoring was used, including none invasive blood pressure, blood oxygen saturation $\left(\mathrm{SpO}_{2}\right)$, electrocardiogram. The surgical time was noted as the time from incision to the end of surgery. On arrival in the Postanesthesia Care Unit (PACU), pain, and other adverse effects such as nausea, vomiting, pruritus, dizziness, hypotension (30 \% reduced from baseline) numbness, and motor blockade were recorded every $15 \mathrm{~min}$. Motor blockade was estimated using a modified Bromage scale $(0=$ no blockade: extended limb lift off the bed; $1=$ flexion/extension at knee and ankle joint; $2=$ no flexion/extension at knee or ankle joint; $3=$ complete blockade). Pain was assessed by visual analog scale (VAS) from 0-10, where $0=$ no pain; $1-3=$ mild pain; 4-7 moderate pain; $8-10=$ severe pain. Tramadol $50 \mathrm{mg}$ intravenous (IV) was given if the VAS $\geq 4$.

Forty eight hours post-operative in the ward, patients in both groups were maintained by continuous infusion of $0.125 \%$ levobupivacaine rate $7 \mathrm{ml} / \mathrm{hr}$ and then reduced to $5 \mathrm{ml} / \mathrm{hr}$ if VAS $\leq 3$. The femoral catheters were removed at $48 \mathrm{~h}$ post-operative. During the hospital stay, all patients received oral ultracet one tab two times a day, oral acetaminophen $500 \mathrm{mg}$ four times a day, and oral lorazepam $0.5 \mathrm{mg}$ before bed time. The patients having breakthrough pain (defined as VAS $\geq 4$ ) were treated on demand with tramadol $50 \mathrm{mg}$ IV every $4 \mathrm{~h}$ until discharge. The blinded residents made visits at 6 , $12,24,36$, and $48 \mathrm{~h}$ post-operative to record adverse effects, pain scores, patients' satisfaction $(1=$ poor, $2=$ fair, 3 = good, and $4=$ excellent). 
Based on the data from Sundarathiti et al. [7], at $12 \mathrm{~h}$ post-operative, $80 \%$ of patients with CFNB after TKA experienced moderate to severe pain, while approximately $38 \%$ of patients with continuous epidural infusion (CEI) did. About 22 patient in each group would suffice to demonstrate a significant difference with a probability of type I error of 0.05 and power value of $80 \%$. The long-term expected rate of patient loss was considered to be about $20 \%$, so finally each group was to have at least 26 patients Detailed information on enrollment of patients into the study is depicted by the CONSORT flow diagram in Fig. 1.

Data were recorded and analyzed using SPSS 15 statistical package (SPSS; Inc., Chicago, IL) for Windows. Results are reported as mean (SD) for continuous variables, where number and percentage for nominal variables. All continuous variables were first checked for normality of data distribution by Shapiro-Wilk test. Independent samples $T$-test or Mann-Whitney $U$ test were used to compare the data between the two study groups depending on data distribution in each measurement. Nominal variables were analyzed by chi-square or Fisher's exact test. A pvalue $<0.05$ was considered statistically significant.
Table 1 Demographic data

\begin{tabular}{llll}
\hline & $\begin{array}{lll}\text { CFNB/SA } \\
(n=33)\end{array}$ & $\begin{array}{l}\text { CFNB/SAMO } \\
(n=35)\end{array}$ & $p$-value \\
\hline Age $(\mathrm{yr})$ & $68.06(8.37)$ & $69.60(7.39)$ & 0.42 \\
Weight $(\mathrm{kg})$ & $59.88(14.42)$ & $62.00(12.72)$ & 0.52 \\
Height $(\mathrm{cm})$ & $154.76(6.19)$ & $155.86(6.94)$ & 0.49 \\
Body mass index (BMI) $\left(\mathrm{kg} / \mathrm{m}^{2}\right)$ & $26.39(5.61)$ & $25.74(4.49)$ & 0.60 \\
ASA (I/II/III) & $0 / 13 / 20$ & $1 / 7 / 27$ & 0.11 \\
Surgical time (mins) & $155.30(34.41)$ & $145.86(39.06)$ & 0.30
\end{tabular}

Data are expressed as number of patients or mean (SD); CFNB/SA Continuous femoral nerve block/Spinal anesthesia; CFNB/SAMO Continuous femoral nerve block/Spinal anesthesia plus Morphine

\section{Results}

A total of 68 patients participated, 33 with spinal anesthesia and femoral catheter, and 35 with the same procedure plus intrathecal morphine. Patient's demographics data (Table 1) in each group were not different. All patients had satisfactory anesthesia and operation without intaoperative complications. There were 2 patients in Group I (CFNB/SA) dropped out from the study due to cognitive changes postoperatively (disorientation to time and place).

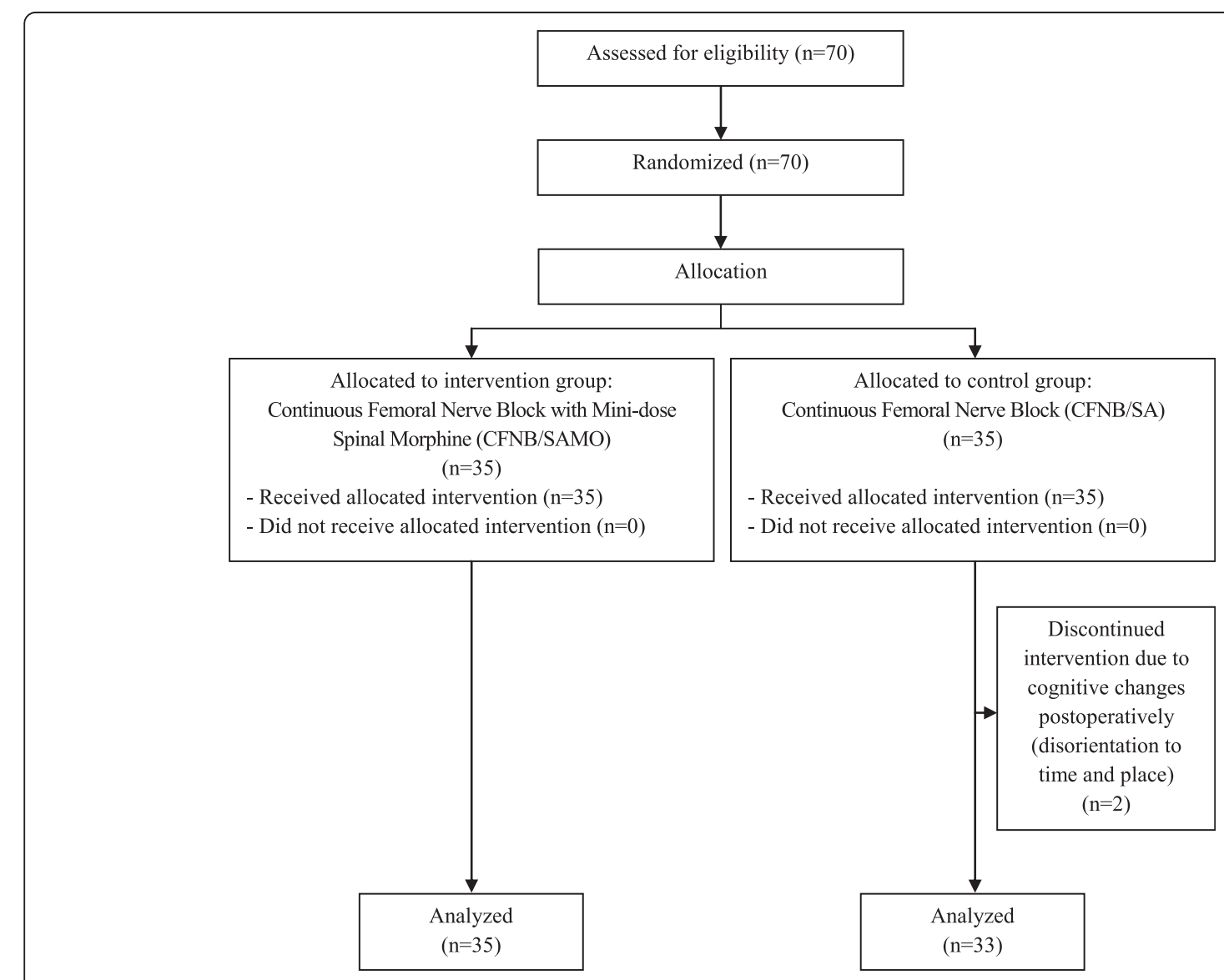

Fig. 1 CONSORT 2010 Flow diagram 
Table 2 Modified Bromage scale (MBS) at 0, 15, 30, 45 and 60 min

\begin{tabular}{|c|c|c|c|c|c|c|c|c|c|c|}
\hline \multirow[t]{3}{*}{ MBS } & \multicolumn{2}{|l|}{0 min } & \multicolumn{2}{|l|}{$15 \mathrm{~min}$} & \multicolumn{2}{|l|}{$30 \mathrm{~min}$} & \multicolumn{2}{|l|}{$45 \mathrm{~min}$} & \multicolumn{2}{|l|}{$60 \mathrm{~min}$} \\
\hline & CFNB/SA & CFNB/SAMO & CFNB/SA & CFNB/SAMO & CFNB/SA & CFNB/SAMO & CFNB/SA & CFNB/SAMO & CFNB/SA & CFNB/SAMO \\
\hline & $(n=33)$ & $(n=35)$ & $(n=33)$ & $(n=35)$ & $(n=33)$ & $(n=35)$ & $(n=33)$ & $(n=35)$ & $(n=33)$ & $(n=35)$ \\
\hline 0 & - & - & - & - & - & - & - & - & - & - \\
\hline 1 & - & - & $2(6.06)$ & - & $3(9.09)$ & $1(2.86)$ & $5(15.15)$ & $5(14.29)$ & $7(21.21)$ & $10(28.57)$ \\
\hline 2 & $7(21.21)$ & $8(22.86)$ & $8(24.24)$ & $16(45.71)$ & $12(36.36)$ & $24(68.57)$ & $20(60.61)$ & 27 (77.14) & $22(66.67)$ & $24(68.57)$ \\
\hline 3 & 26 (78.79) & $27(77.14)$ & $23(69.70)$ & 19 (54.29) & $18(54.55)$ & $10(28.57)$ & $18(24.24)$ & $3(8.57)$ & $4(12.12)$ & $1(2.86)$ \\
\hline$p$-value & 0.87 & & 0.29 & & 0.10 & & 0.27 & & 0.23 & \\
\hline
\end{tabular}

The values are expressed as number of patients (percent)

MBS modified bromage scale $(0=$ no blockade; $1=$ for flexion/extension at knee and ankle joint; $2=$ no flexion/extension at knee and ankle joint; and 3 = complete blockade)

Residual motor blockade was similar in both group (Table 2).

The VAS scores and the number of patients suffering from 'moderate to severe pain' are presented in Tables 3 and 4. Experiencing pain was significantly less pronounced in patients with spinal morphine (CFNB/SAMO), starting $6 \mathrm{~h}$ after surgery during the entire investigation period; these differences were significant at $6,12,24$, and $48 \mathrm{~h}$ respectively. Cumulative tramadol IV requirement was significantly lesser in the CFNB/SAMO group (median $=125 \mathrm{mg}$, range $50-400 \mathrm{mg}$ ) compared with the $\mathrm{CFNB} / \mathrm{SA}$ group (median $=200 \mathrm{mg}$, range $50-500 \mathrm{mg}$ ) as presented in Fig. $2(p$-value $=0.01)$. As presented in Fig. 3 , at $6 \mathrm{~h}$ after surgery a significant higher rate of CFNB/SAMO patients were affected by PONV (approx. 40 vs. $15 \%$; $p<$ 0.05). Patients in both groups had similar overall incidences of other side effect such as pruritus, dizziness and hypotension ( $p$-value $>0.99$ in all side effects) except the incidences of numbness during PO1-6 h which was significantly greater in the CFNB/SAMO group (p-value 0.03 and 0.04 respectively). Patient's satisfaction rated as good or excellent was not different between the two groups.

\section{Discussion}

In this study, we used a very small dose of intrathecal morphine $0.035 \mathrm{mg}$ in addition to femoral block (CFNB/ SAMO) to complete the analgesia without any more

Table 3 Median (range) of VAS scores

\begin{tabular}{llll}
\hline & $\begin{array}{l}\text { CFNB/SA } \\
(n=33)\end{array}$ & $\begin{array}{l}\text { CFNB/SAMO } \\
(n=35)\end{array}$ & $p$-value \\
\hline Postoperative hr & VAS $(1-10)$ & & \\
1 & $0(0-2)$ & 0 (constant) & 0.14 \\
6 & $5(0-10)$ & $3(0-9)$ & 0.05 \\
12 & $6(0-10)$ & $3(0-7)$ & $<0.01$ \\
24 & $5(0-10)$ & $3(0-7)$ & $<0.01$ \\
36 & $3(0-10)$ & $2(0-8)$ & 0.18 \\
48 & $4(0-10)$ & $3(0-7)$ & 0.03 \\
\hline
\end{tabular}

Data are shown as median (min-max) effort and care on the side of the anesthesiologists. The pain scores (VAS) 6, 12, 24 and $48 \mathrm{~h}$ after surgery and the cumulative tramadol IV requirement (Fig. 2) were significantly lesser in the CFNB/SAMO group compared with the CFNB/SA group. The VAS scores at PO1-6 $\mathrm{h}$ were not different, which may be partly due to the residual analgesia after spinal anesthesia. Whereas motoric function was not affected by the respective method, the rate of PONV was signifivantly higher in patients with morphine at the $6^{\text {th }}$ hour after surgery (approx. 40 vs. $15 \%$ ) but not any further (Fig. 3).

The effect of CFNB in this study was not satisfactory, because the VAS scores in both groups were quite high (Table 3). Categorizing VAS scores $\geq 4$ as 'failure' we observed a failure rate between 6 and 48 h postoperatively of $39.4-69.7 \%$ and $31.4-48.6 \%$ in patients with CFNB and patients with CFNB plus spinal morphine respectively (Table 4). Pöpping et al. ${ }^{9}$ in their large observational study including 1374 patients with femoral/ sciatic block had a failure rate (no analgesic effect) of $3.96 \%$ and a rate of malposition after correct placement of $15.2 \%$. Among others we have to contemplate the possibility of secondary block failure, where the catheter has not been positioned appropriately. Although the block was achieved with the stimulation technique, the trigger during stimulation applied in this study with < $0.5 \mathrm{~mA}$ was probably too high. In addition the initial

Table 4 Incidence of moderate to severe pain (VAS 4-7)

\begin{tabular}{llll}
\hline & $\begin{array}{l}\text { CFNB/SA } \\
(n=33)\end{array}$ & $\begin{array}{l}\text { CFNB/SAMO } \\
(n=35)\end{array}$ & $p$-value \\
\hline Postoperative, hr & Patients, $\mathrm{n}(\%)$ & & - \\
1 & - & - & 0.13 \\
6 & $22(66.67 \%)$ & $17(48.57 \%)$ & 0.03 \\
12 & $23(69.70 \%)$ & $15(42.86 \%)$ & 0.08 \\
24 & $22(66.67 \%)$ & $16(45.71 \%)$ & 0.49 \\
36 & $13(39.39 \%)$ & $11(31.43 \%)$ & 0.03 \\
48 & $17(51.52 \%)$ & $9(25.71 \%)$ &
\end{tabular}

Data are shown as number and percentage 


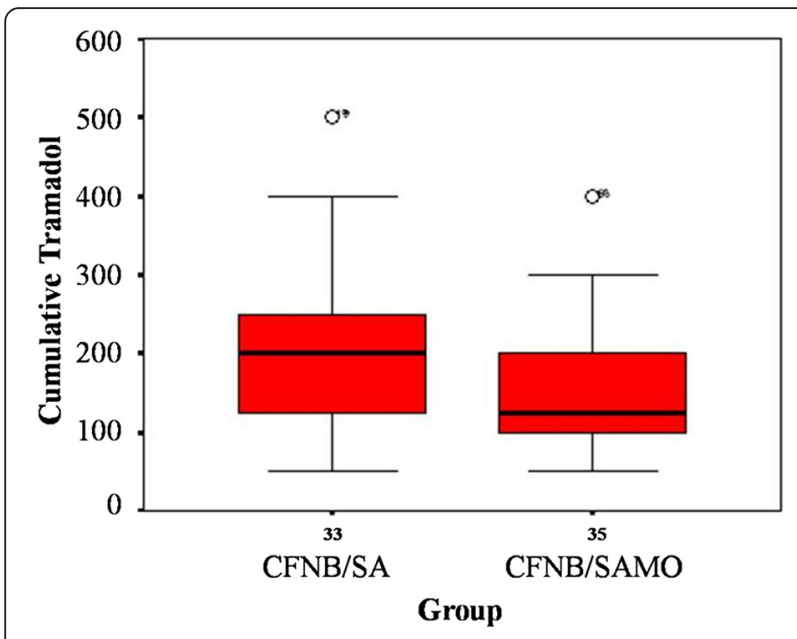

Fig. 2 Cumulative tramadol IV requirement for PO48h

dose of local anesthetics might have been too low and unnoticed catheter migration could also lead to failure. Inadequate efficacy of FNB alone for knee surgery is due to the fact that the posterior part of the knee is innervated by the sciatic nerve. Hence sciatic block has been added to FNB by many study groups $[8,9,12-14]$, though it is unclear whether blocking both the femoral and sciatic nerve may result in a greater risk of direct needle trauma to the peripheral nerve. Performance of sciatic nerve block is time-consuming, requiring additional costs and skill. However, our data clearly indicate that femoral block alone is not sufficient in major knee surgery, as it does not affect the popliteal area, which is in accordance to Sundarathiti $P$ et al. [7] reporting inferior analgesic efficacy of FNB compared with epidural analgesia.
The purpose of our study was to find a simple 'multimodal' form of postoperative pain control, using a technique that can be added to femoral catheter in patients with major knee surgery under spinal anesthesia. Multimodal analgesia combines alternative strategies with the goal to avoid routine parenteral narcotics, and minimize the side effects of the respective method [15-18]. It takes advantage of the synergistic effects of various analgesics, permitting the use of smaller doses.

Intrathecal opioids added to local anesthetics during spinal anesthesia have been applied in a variety of surgical settings since 1979 [19], providing prolonged postoperative analgesia without the need for catheters or expensive pumps. However, the use of intrathecal morphine may be associated with distressing side effects, such as itching, urinary retention, nausea and vomiting (PONV), and respiratory depression [20]. In an attempt to limit opioid side effects, the use of low-dose spinal opioids has been advocated [21]. Even mini-dose morphine $(<0.1 \mathrm{mg}$ ) was frequently reported to be effective for managing acute postoperative pain after variety of surgeries without any evidence of respiratory depression [22, 23].

Achieving high quality pain relief after TKA is possible using regional anesthesia and multimodal pain management [24]. In patients already treated with spinal anesthesia intrathecal opioid analgesia (ITOA) has specific advantages regarding ease of administration, safe and rapid onset of action and low costs. A single dose administered at the time of surgery can provide good neuroaxial analgesia during the first postoperative day. Because spinal morphine is frequently accompanied by a high rate of PONV and itching, we tried a very low dose with $0.035 \mathrm{mg}$ and found a temporary increase in

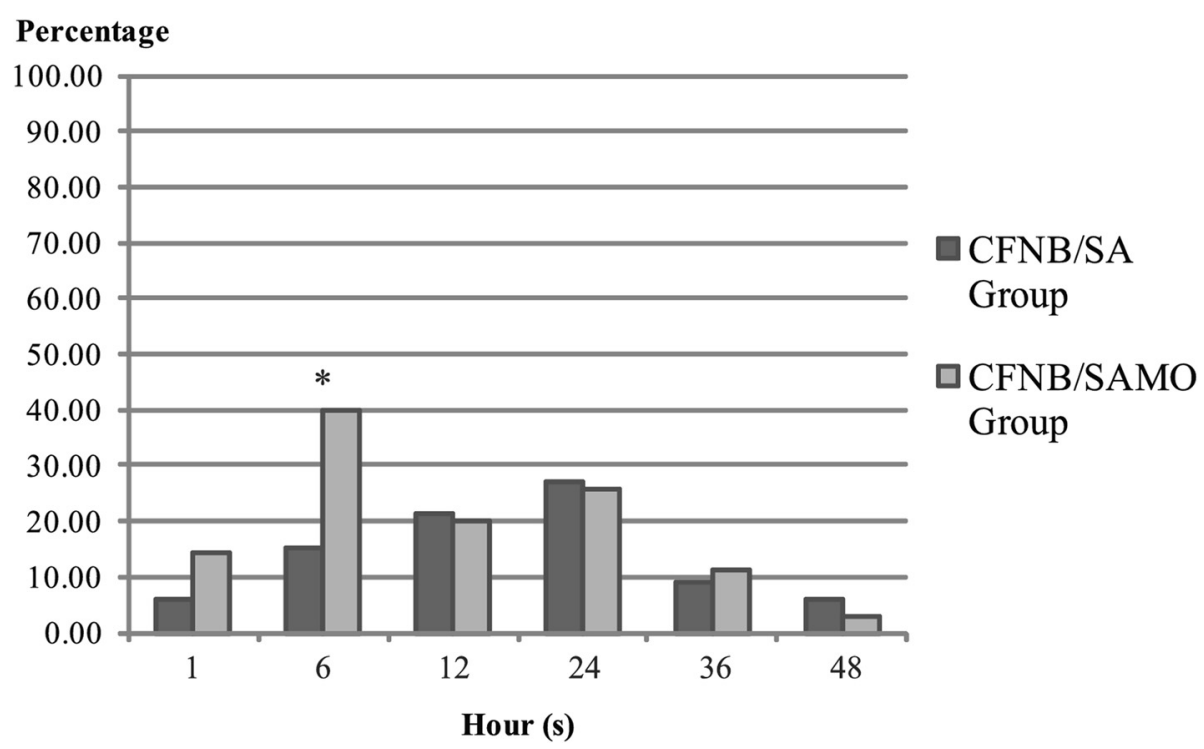

Fig. 3 Incidence of nausea/vomiting 
numbness which was clinically irrelevant, but a significant increase in PONV $6 \mathrm{~h}$ after surgery. There were no further or late side effects. We can state mini-dose intrathecal morphine (ITMO) is a safe, effective, and relatively inexpensive modality for the management of postoperative pain. We estimate CFNB and mini-dose ITMO as a good combination-technique to achieve the goal of multimodal pain management for TKA.

\section{Conclusion}

Postoperative analgesia with continuous femoral block (CFNB) alone for total knee arthroplasty (TKA) is inadequate. A mini-dose of intrathecal morphine in addition to CFNB was found to be simple, safe, inexpensive and more effective than CFNB alone for managing postoperative pain after TKA with little side effects. However, the analgesic effect could be better. Future studies have to determine the most appropriate dose of spinal morphine when added to proper working femoral analgesia.

\section{Abbreviations \\ ASA, American Society of Anesthesiologists; BMI, body mass index; CFNB, continuous femoral nerve block; CFNB/SAMO, continuous femoral nerve block with mini-dose spinal morphine; $\mathrm{Cr}$, creatinine level; EA, epidural analgesia; ITMO, intrathecal morphine; ITOA, intrathecal opioid analgesia; IV, intravenous; PACU, post anesthesia care unit; PNB, peripheral nerve block; $\mathrm{PO}$, post-operative; PONV, post-operative nausea and vomiting; SA, spinal anesthesia; TKA, total knee arthroplasty; VAS, visual analog scale.}

\section{Acknowledgement}

We would like to thank Prof. Benno von Bormann, Siriraj Hospital, Bangkok for his kind consultation in this study and Miss Rojnarin Komonhirun for her statistical advice.

\section{Funding}

This study was supported by Research Grant from the Faculty of Medicine Ramathibodi Hospital, Mahidol University No. 53088.

\section{Availability of supporting data}

No supporting data.

\section{Authors' contributions}

PS is the principal investigator who directed the study and wrote the manuscript. JT helped to write the manuscript. SSu performed data analysis and interpretation. SSa obtained the consent of participants and collected the data. MP collected the data. All authors read and approved the final manuscript.

\section{Competing interests}

The authors declare that they have no competing interests.

\section{Consent for publication}

Not applicable.

\section{Ethics approval and consent to participate}

Ethics approval was approved by the Ethical Committee, Ramathibodi Hospital. Each participant has given written informed consent before participating in the study.

Received: 9 July 2015 Accepted: 1 June 2016

Published online: 16 July 2016

\section{References}

1. Capdevila X, Barthelet Y, Biboulet P, Ryckwaert Y, Rubenovitch J, d'Athis F. Effects of perioperative analgesic technique on the surgical outcome and duration of rehabilitation after major knee surgery. Anesthesiology. 1999;91(1):8-15.
2. Singelyn FJ, Deyaert M, Joris D, Pendeville E, Gouverneur JM. Effects of intravenous patient-controlled analgesia with morphine, continuous epidural analgesia, and continuous three-in-one block on postoperative pain and knee rehabilitation after unilateral total knee arthroplasty. Anesth Analg. 1998;87(1):88-92.

3. Wang $\mathrm{H}$, Boctor B, Verner J. The effect of single-injection femoral nerve block on rehabilitation and length of hospital stay after total knee replacement. Reg Anesth Pain Med. 2002;27(2):139-44.

4. Bonica J. Postoperative pain. In: Bonica J, editor. The Management of Pain. 2nd ed. Philadelphia: Lea \& Febiger; 1990. p. 461-80.

5. Pang WW, Hsu TC, Tung CC, Hung CP, Chang DP, Huang MH. Is total knee replacement more painful than total hip replacement? Acta Anaesthesiol Sin. 2000;38(3):143-8.

6. Fowler SJ, Symons J, Sabato S, Myles PS. Epidural analgesia compared with peripheral nerve blockade after major knee surgery: a systematic review and meta-analysis of randomized trials. Br J Anaesth. 2008;100(2):154-64.

7. Sundarathiti P, Ruananukul N, Channum T, Kitkunasathean C, Mantay A, Thammasakulsiri J, et al. A comparison of continuous femoral nerve block (CFNB) and continuous epidural infusion (CEI) in postoperative analgesia and knee rehabilitation after total knee arthroplasty (TKA). J Med Assoc Thai. 2009;92(3):328-34.

8. Bauer MC, Pogatzki-Zahn EM, Zahn PK. Regional analgesia techniques for total knee replacement. Curr Opin Anaesthesiol. 2014;27:501-6.

9. Pöpping DM, Zahn PK, Van Aken HK, et al. Effectiveness and safety of postoperative pain management: a survey of 18925 consecutive patients between 1998 and 2006 (2nd revision): a database analysis of prospectively raised data. Br J Anaesth. 2008;101:832-40.

10. Abdallah FW, Brull R. Is sciatic nerve block advantageous when combined with femoral nerve block for postoperative analgesia following total knee arthroplasty? Reg Anesth Pain Med. 2011;36:493Y498.

11. Tierney E, Lewis G, Hurtig JB, Johnson D. Femoral nerve block with bupivacaine $0.25 \%$ for postoperative analgesia after open knee surgery. Can J Anaesth. 1987;34(5):455-8.

12. Weber A, Fournier R, Van Gessel E, Gamulin Z. Sciatic nerve block and the improvement of femoral nerve block analgesia after total knee replacement. Eur J Anaesthesiol. 2002;19(11):834-6.

13. Mansour NY, Bennetts FE. An observational study of combined continuous lumbar plexus and single-shot sciatic nerve blocks for post-knee surgery analgesia. Reg Anesth. 1996;21(4):287-91.

14. Chelly JE, Greger J, Gebhard R, Coupe K, Clyburn TA, Buckle R, et al. Continuous femoral blocks improve recovery and outcome of patients undergoing total knee arthroplasty. J Arthroplasty. 2001;16(4):436-45.

15. Joshi GP. Multimodal analgesia techniques for ambulatory surgery. Int Anesthesiol Clin. 2005;43(3):197-204.

16. Maheshwari AV, Boutary M, Yun AG, Sirianni LE, Dorr LD. Multimodal analgesia without routine parenteral narcotics for total hip arthroplasty. Clin Orthop Relat Res. 2006;453:231-8.

17. Parvataneni HK, Shah VP, Howard H, Cole N, Ranawat AS, Ranawat CS. Controlling pain after total hip and knee arthroplasty using a multimodal protocol with local periarticular injections: a prospective randomized study. J Arthroplasty. 2007;22(6 Suppl 2):33-8.

18. Peters $\mathrm{CL}$, Shirley $\mathrm{B}$, Erickson J. The effect of a new multimodal perioperative anesthetic regimen on postoperative pain, side effects, rehabilitation, and length of hospital stay after total joint arthroplasty, J Arthroplasty. 2006;21(6 Suppl 2):132-8.

19. Wang JK, Nauss LA, Thomas JE. Pain relief by intrathecally applied morphine in man. Anesthesiology. 1979;50(2):149-51.

20. Chinachoti T1, Nilrat P, Samarnpiboonphol P. Nausea, vomiting and pruritus induced by intrathecal morphine. J Med Assoc Thai. 2013;96(5):589-94.

21. Suksompong $S$, Pongpayuha $P$, Lertpaitoonpan W, von Bormann B, Phanchaipetch T, Sanansilp V. Low-dose spinal morphine for postthoracotomy pain: a prospective randomized study. J Cardiothorac Vasc Anesth. 2013;27:417-22.

22. Kalso E. Effects of intrathecal morphine, injected with bupivacaine, on pain after orthopaedic surgery. Br J Anaesth. 1983;55(5):415-22.

23. Kirson LE, Goldman JM, Slover RB. Low-dose intrathecal morphine for postoperative pain control in patients undergoing transurethral resection of the prostate. Anesthesiology. 1989;71(2):192-5.

24. Iffeld BM. Continuous peripheral nerve blocks: a review of the published evidence. Anesth Analg. 2011;113:904-25. 